\title{
ON THE REGULARITY OF BOUNDARY POINTS IN POTENTIAL THEORY
}

\author{
H. L. ROYDEN
}

1. Let $G$ be a bounded region (open connected set) in three-dimensional Euclidean space, and let $f(P)$ be a function which is defined and continuous on the boundary $R$ of $G$. Then the problem of Dirichlet is to find a function $u$ which is harmonic in $G$ and which satisfies

$$
\lim _{Q \rightarrow P} u(Q)=f(P), \quad P \in R .
$$

A unique harmonic function $u$ may be constructed from the given boundary values by any of several methods, for example, by exhausting $G$ with a sequence of domains for which the Dirichlet problem is solvable [3], ${ }^{1}$ by using subharmonic functions [4], or by the Dirichlet principle $[1 ; 5]$. There then arises the question of whether the relation (1) holds for our function $u$ or not. This leads us to the following definition: A point $P_{0}$ of $R$ is said to be regular for the Dirichlet problem if for every set of continuous boundary values $f(P)$ we always have

$$
\lim _{Q \rightarrow P_{0}} u(Q)=f\left(P_{0}\right)
$$

A well known condition due to Poincare is that the point $P_{0}$ is regular if there is a cone with vertex at $P_{0}$ having no points in common with $G$. Another sufficiency condition, also due to Poincaré, is that $P_{0}$ shall belong to an ellipse which has no points in common with $G$ [3]. On the basis of recent work by Evans [2], one may also show that $P_{0}$ is regular if there is a triangle with a vertex at $P_{0}$ which does not meet $G$.

In the present paper we prove the following sufficiency condition for regularity which includes the above three conditions.

TheOREM. $A$ point $P_{0}$ of $R$ is regular if there is some sphere ${ }^{2} S$ with center at $P_{0}$ such that the points of $S$ which can be joined to $P_{0}$ by radial segments exterior to $G$ form a set $E^{*}$ of positive logarithmic capacity.

We may take the logarithmic capacity of $E^{*}$ to be the logarithmic

Received by the editors January 22, 1951.

1 Numbers in brackets refer to the bibliography at the end of the paper.

${ }^{2}$ By a sphere we mean the surface of a sphere. By interior of a sphere is meant the region surrounded by the sphere. 
capacity of the plane set $E$ formed from $E^{*}$ by stereographic projection from some point $Q_{1}$ on $S$ which is in $G$, since the capacity of this set vanishes if and only if the usual logarithmic capacity of $E^{*}$ vanishes [6]. In the cases where we have a cone of a triangle touching $P_{0}$, the sets $E$ contain a circle or a line segment respectively, and hence have positive logarithmic capacity.

2. In order to prove this theorem, we shall need two lemmas concerning plane regions, which we now proceed to prove.

LEммA I. Let the plane set $E$ lying in $|z| \leqq M$ have logarithmic capacity $C$. Then the Green's function $G(z, \zeta)$ of the region complementary to $E$ satisfies the following inequality

$$
G(z, \zeta) \leqq \log \frac{(M+|z|)(M+|\zeta|)}{C|z-\zeta|} .
$$

Proof. The function

$$
h(z)=G(z, \zeta)-G(z, \infty)+\log |z-\zeta|
$$

is harmonic in the complement of $E$. We have $G(z, \zeta)=0$ for $z \in E$ except for a set of capacity zero [6], while on $E$ we have

$$
\log |z-\zeta| \leqq \log (M+|\zeta|)
$$

and

$$
G(z, \infty) \geqq 0 .
$$

Hence by the maximum principle

$$
h(z) \leqq \log (M+|\zeta|)
$$

in the complement of $E$. Thus we have

$$
G(z, \zeta)-G(z, \infty)+\log |z-\zeta| \leqq \log (M+|\zeta|),
$$

whence

$$
G(z, \zeta) \leqq G(z, \infty)-\log |z-\zeta|+\log (M+|\zeta|) .
$$

Near the point $z=\infty, G(z, \infty)$ has the expansion

$$
G(z, \infty)=\log |z|-\log C+O\left(\frac{1}{|z|}\right) .
$$

Hence, letting $z$ tend to $\infty$ in (3), we get

$$
G(\zeta, \infty)=G(\infty, \zeta) \leqq-\log C+\log (M+|\zeta|) .
$$


Using this to estimate $G(z, \infty)$ in (3), we get $G(z, \zeta) \leqq-\log C+\log (M+|z|)-\log |z-\zeta|+\log (M+|\zeta|)$, from which (2) follows.

LEMma II. For some $\beta>0$, there is a function $v(z)$ which is defined, twice continuously differentiable, and greater than unity in the complement of $E$, and which satisfies there the differential equation

$$
\nabla^{2} v+\frac{2 \pi \beta}{\left(1+|z|^{2}\right)^{2}} v=0 .
$$

Proof. Let

$$
\mu_{k}(z)=\iint_{C(E)} \frac{G(z, \zeta)}{\left(1+|\zeta|^{2}\right)^{2}} \mu_{k-1}(\zeta) d \omega_{\zeta},
$$

where $\mu_{0}(z) \equiv 1$. Set $m_{k}=\sup \mu_{k}(z)$ for $z \in C(E)$. Then

$$
\mu_{k}(z) \leqq m_{k-1} \iint_{C(E)} \frac{G(z, \zeta)}{\left(1+|\zeta|^{2}\right)^{2}} d \omega_{\zeta}
$$

But by Lemma I,

$$
\begin{aligned}
\iint_{C(E)} \frac{G(z, \zeta)}{\left(1+|\zeta|^{2}\right)^{2}} & d \omega_{\zeta} \\
& \leqq \iint \log \frac{(M+|z|)(M+|\zeta|)}{C|z-\zeta|} \frac{d \omega}{\left(1+|\zeta|^{2}\right)^{2}}
\end{aligned}
$$

while the latter integral is, by elementary calculation, less than $2 \pi\left(|\log C|+\log 150+\left|\log M^{2}\right|\right)=A$. Thus $\mu_{k}(z) \leqq A^{k}$, and for $\beta$ $\leqq 1 / 2 A$ the series

$$
v(z)=2+2 \sum_{k=1}^{\infty} \beta^{k} \mu_{k}(z)
$$

converges uniformly in $C(E)$ and is greater than one there. Thus

$$
\beta \iint_{C(E)} \frac{G(z, \zeta) v(\zeta)}{\left(1+|\zeta|^{2}\right)^{2}} d \omega_{\zeta}=v(z)-2
$$

whence $v(z)$ possesses continuous second derivatives and

$$
-\frac{1}{2 \pi} \nabla^{2} v(z)=\beta \frac{v(z)}{\left(1+|z|^{2}\right)^{2}},
$$

so that $v(z)$ satisfies the requirements of the lemma. 
3. We now proceed to prove our theorem. It is well known $[4, \mathrm{p}$. 51] that the point $P_{0}$ is regular if we can find a neighborhood $U$ of $P_{0}$ in which there is a barrier for $P_{0}$, that is, a function $u(Q)$ which is defined in $U \cap G$ and satisfies

(i) $u(Q)$ is harmonic in $U \cap G$;

(ii) $u\left(P_{0}\right)=0$;

(iii) lim inf $u(Q)>0$ as $Q \rightarrow P \neq P_{0}$ on the boundary of $U \cap G$.

Let $U$ be the interior of $S$, and let $u$ be a function which is defined in the region formed by the interior of $S$ less the segments from $P_{0}$ to $E^{*}$. Suppose $u=R^{\alpha} v(\theta, \phi)$, with $\alpha>0$, where $R, \theta, \phi$ are the spherical coordinates with origin at $P_{0}$ and with polar axis through $Q_{1}$. Then if we can choose a $v(\theta, \phi) \geqq 1$ so that $u$ is harmonic, the function $u$ will be a barrier for $P_{0}$. But if $u$ is harmonic, we have

$$
\begin{aligned}
0=\nabla^{2} u \equiv & \frac{1}{R^{2}} \frac{\partial}{\partial R}\left(R^{2} \frac{\partial u}{\partial R}\right)+\frac{1}{R^{2} \sin \theta} \frac{\partial}{\partial \theta}\left(\sin \theta \frac{\partial u}{\partial \theta}\right) \\
& +\frac{1}{R^{2} \sin ^{2} \theta} \frac{\partial^{2} u}{\partial \phi^{2}},
\end{aligned}
$$

whence $v$ must satisfy

$$
\alpha(\alpha+1) v+\frac{1}{\sin \theta} \frac{\partial}{\partial \theta}\left(\sin \theta \frac{\partial v}{\partial \theta}\right)+\frac{1}{\sin ^{2} \theta} \frac{\partial^{2} v}{\partial \phi^{2}}=0 .
$$

Letting $\rho=\operatorname{ctg} \theta / 2$, we have $\sin \theta=2 \rho /\left(1+\rho^{2}\right)$, and $\sin \theta \partial v / \partial \theta$ $=\partial v / \partial \rho$, so that

$$
\alpha(\alpha+1) v+\frac{\left(1+\rho^{2}\right)^{2}}{\rho} \frac{\partial}{\partial \rho}\left(\rho \frac{\partial v}{\partial \rho}\right)+\frac{\left(1+\rho^{2}\right)^{2}}{\rho^{2}} \frac{\partial^{2} v}{\partial \phi^{2}}=0,
$$

or

$$
\nabla_{z}^{2} v+\frac{2 \pi \beta}{\left(1+|z|^{2}\right)^{2}} v=0,
$$

where we have set $z=\rho e^{i \phi}$. Since the transformation $(\theta, \phi)$ to $(\rho, \phi)$ is the stereographic projection of $S$ onto the $z$-plane, $v$ needs to be defined only outside the set $E$. But the existence of such a function $v$ in the complement of $E$ is assured us by Lemma II.

\section{BIBLIOGRAPHY}

1. R. Courant and D. Hilbert, Methoden der mathematische Physik, vol. 2, Berlin, 1937, chap. 7.

2. G. C. Evans, Lectures on multiple valued harmonic functions in space, University of California Publications in Mathematics, vol. 1, no. 8, pp. 281-340. 
3. O. D. Kellogg, Foundations of potential theory, Berlin, 1929.

4. O. Perron, Eine neue Behandlung der ersten Randwertaufgabe fiur $\Delta u=0$, Math. Zeit. vol. 18 (1923).

5. H. Weyl, The method of orthogonal projection in potential theory, Duke Math. J. vol. 7 (1940).

6. O. Frostman, Potential d'equilibre et capacite des ensembles avec quelques applications d la theorie des fonctions, Meddel. Lunds Univ. Mat. Sem. vol. 3 (1935).

HARVARD UNIVERSITY

\section{A NOTE ON AREA ${ }^{1}$}

\section{EDWARD SILVERMAN}

If $\bar{x}$ is continuous on a square $Q$ into a metric space $D$, then it is known that there exists a continuous function $x$ on $Q$ into $m$, the space of bounded sequences, which is isometric to $\bar{x}[1] .{ }^{2}$ By an area let us understand a functional on the set of continuous functions on $Q$ into $m$ which has the property that if $\bar{x}$ maps $Q$ into $E_{3}$, if $\bar{x}$ is sufficiently smooth, and if $x$ on $Q$ into $m$ is isometric with $\bar{x}$, then the area of $x$ agrees with the generally accepted area of $\bar{x}$.

If $x$ and $y$ map $Q$ into $m$ and if $\|x(p)-x(q)\| \leqq\|y(p)-y(q)\|$ for all $p$ and $q$ in $Q$, then let us write $x<y$.

Let $\mu$ be a measure on $m$ such that if $G \subset m, G$ is isometric to $H$, $H \subset E_{3}$, then the $\mu$-measure of $G$ is equal to the 3-dim Lebesgue measure of $H$.

Suppose that it is considered reasonable to impose upon an area the following (Kolmogoroff's) principle: if $x<y$, then area $x \leqq$ area $y$. We shall see, then, that an area must suffer from one, at least, of the following two maladies:

(i) There exists a function whose area is finite and whose range has positive $\mu$-measure.

(ii) There exists a function whose area is infinite and whose range is a (simply covered) simple arc.

It is sufficient to show that an area $\alpha$ which satisfies Kolmogoroff's principle and is not subject to (i) must necessarily satisfy (ii).

Let $x$ be continuous on $Q$ into $m$. It is not difficult to construct a monotone function $x^{\prime}$ with $x<x^{\prime}$ such that $x^{\prime}$ is the monotone factor

Received by the editors February 19, 1951.

${ }_{1}^{1}$ The author is grateful for financial support from the Research Corporation.

${ }^{2}$ Numbers in brackets refer to the bibliography at the end of the paper. 